\title{
Revisión de educación en valores para el nivel superior en Latinoamérica
}

\author{
Moreno Jácome, María Cristina; Ramírez Ramírez, Leticia Nayeli; Escobar Pérez, José Zenón \\ Revisión de educación en valores para el nivel superior en Latinoamérica \\ Revista Educación, vol. 44, núm. 1, 2020 \\ Universidad de Costa Rica, Costa Rica \\ Disponible en: http://www.redalyc.org/articulo.oa?id=44060092008 \\ DOI: https://doi.org/10.15517/revedu.v44i1.35636
}

Esta obra está bajo una Licencia Creative Commons Atribución-NoComercial-SinDerivar 3.0 Internacional. 


\section{Revisión de educación en valores para el nivel superior en Latinoamérica}

Review of Values Education for Higher Education in Latin America

Maria Cristina Moreno Jácome

Instituto Politécnico Nacional, México

mmorenoj@ipn.mx

(iD) http://orcid.org/0000-0003-1754-3282

Leticia Nayeli Ramírez Ramírez

Universidad Panamericana, Escuela de Pedagogía., México

nramirez@up.edu.mx

iD http://orcid.org/0000-0002-8113-2368

José Zenón Escobar Pérez

Universidad Autónoma de México, México

zenon_dgeta@hotmail.com

iD http://orcid.org/0000-0002-0059-9792
DOI: https://doi.org/10.15517/revedu.v44i1.35636

Redalyc: http://www.redalyc.org/articulo.oa?id=44060092008
Recepción: 05 Mayo 2019

Aprobación: 20 Agosto 2019

\section{Resumen:}

La presente revisión sistemática de literatura (SLR) pretende abordar e identificar la producción académica en la temática de educación en valores para la Educación Superior del siglo XXI, donde se verifica la necesidad de basar la educación en valores en el contexto actual. Esta investigación, toma como punto de partida la indagación internacional a través de la revisión sistemática en bases de datos Dialnet, Redalyc y Scielo; se localizaron un total de 35 artículos de países de Latinoamérica mediante un proceso de clarificación y con cuatro criterios de inclusión: 1. Búsqueda de estudios en bases de datos en Dialnet, Redalyc y Scielo; 2. Pertenecientes al campo educación en valores en el nivel superior; 3. Investigaciones desarrolladas en los periodos 2013 al 2018 , 4. De acceso abierto, se conformó una muestra final de 14 trabajos de investigación. Los hallazgos sostienen la participación de las Instituciones de Educación Superior (IES) para replantear su propósito y compromiso con la sociedad para la formación sólida de profesionistas con valores éticos y morales, la preparación del cuerpo docente que emplee una pedagogía orientada a la buena toma de decisiones y actitudes reflexivas apoyándose de un diseño de escala valoral acorde a las necesidades y contexto económico y social en el alba del siglo XXI, así mismo; se desate la ejecución de políticas educativas orientadas a la educación en valores. Se sugiere para futuras investigaciones la publicación de resultados referentes a la implementación y ejecución de propuestas valorales que los autores sitúan como válidas para el contexto actual.

Palabras Clave: Políticas educativas, Educación superior, Educación en valores, Escala de valores y Formación docente.

\section{Abstract:}

This Systematic Literature Review (SLR) aims to address and identify academic progress in the subject of Values Education at Higher Education Institutions in the XXIst century and adapt it today's context. The point of departure for this international study was based on a systematic database review (Dialnet, Redalyc and Scielo); A total number of 35 articles from Latin America were found based on the following four inclusion criteria: 1 . Database Search: Dialnet, Redalyc and Scielo, 2. Fields that included the term Values Education; 3. Studies that covered periods ranging from 2013 - 2018 and, 4. Open access. A final sample of 14 research papers was collected. According to our findings, Higher Education Institutions (HEI) must rethink their purpose and commitment to society in order to educate students with solid ethical and moral values. Faculty requires more training in employing pedagogy skills aimed at teaching good critical thinking and decision-making skills based on a values scale consistent with XXIst century needs and economic and social contexts, directed at enacting government policies in values education. It is suggested that future research focus on implementing and proposing values considered as valid for today's context.

KEYWORDs: Educational Policies, Higher Education, Education in Values, Scale of Values and Teacher Training. 


\section{INTRODUCCIÓN}

El presente estudio permitió desarrollar un análisis de la educación en valores, un tema importante como se ha venido instituyendo y reconociendo por la Organización de las Naciones Unidas para la Educación, la Ciencia y la Cultura, UNESCO (1998) a través de la Declaración Mundial sobre la Educación Superior en el siglo XXI, en el umbral de un nuevo milenio, la educación superior está llamada a conseguir que prevalezcan los valores; entonces se reconoce la labor y el compromiso que tienen las Instituciones de Educación Superior (IES), para direccionar esfuerzos y enfocarse en el desarrollo integral de los estudiantes universitarios con un enfoque humanista.

La educación del siglo XXI se encuentra en una fase de transformaciones sustantivas derivadas de los cambios acelerados que trae consigo los efectos de la globalización, donde la información es de todos y para todos por la disposición que tiene de estar presente en la interconectividad. Estos cambios vertiginosos, han generado consecuencias relacionadas con la educación superior al ofertar a través de planes y programas de estudio conocimientos los cuales se abocan en temas de desarrollo científico y tecnológico, con el objetivo de atender las necesidades y demandas de un mercado laboral altamente competitivo y globalizado, dejando de lado el desarrollo del ser humano integral que toda persona necesita adquirir para la convivencia Moreno y Ramírez (2018).

Es significativo señalar que, a través de datos estadísticos de la Organización para la Cooperación y el Desarrollo Económicos OCDE (2017) quien informa que el 17\% de los jóvenes de entre 25 a 64 años en México había cursado la educación superior en el 2016, la proporción más baja entre los países de la OCDE. Esto es 20 puntos porcentuales abajo del promedio de la OCDE (37\%), pero más alto que en algunos países socios, tales como Brasil (15\%), China (10\%), India (11\%), Indonesia (10\%) y Sudáfrica (12\%).

Estos datos reflejan las realidades que se viven, como se aprecia en la OCDE es muy baja la cantidad de estudiantes formados en educación superior para el año 2016, lo cual significa que existen diversas razones por las que jóvenes no cursan este nivel; en ello es pertinente considerar que una de esas causas es que se necesita del apoyo de valores presentes desde el hogar, dado que el primer contacto social es la familia y es el eje fundamental para la educación en valores; y es a través del apoyo de los valores que los jóvenes se encuentren motivados para estudiar y vencer las dificultades.

Como lo señala Merino (2007) la importancia que cobra la familia en esta formación moral inicial de la persona es tanto más significativa por cuanto, los valores y virtudes se transmiten y desarrollan principalmente por medio de modelos concretos, a través de la identificación emocional con determinadas figuras familiares. Como se puede apreciar, la familia es referencia de vida y trascendental en el establecimiento de valores y con ello la motivación para afrontar los retos de la vida, y uno de ellos está relacionado con el porvenir y desarrollo profesional a través de una formación integral en la educación superior.

Otro dato característico de acuerdo con las estadísticas que anuncia la Secretaría de Educación Pública [SEP] (2017) las Principales Cifras (2017-2018) informan que en México existen cerca de 4.412 escuelas de nivel superior en modalidad escolarizada; y cerca de 325.136 docentes universitarios forman a 3.536.340 alumnos, que se incorporaran al sector productivo aplicando conocimientos y valores obtenidos en la educación superior.

Este contexto impulsa a que las IES lleven a cabo estrategias pedagógicas apoyadas por planes y programas de estudio los cuales deben responder a los retos del siglo XXI, al desarrollar en las personas los valores que requieren para la vida. El respeto y el apoyo al quehacer de los maestros, esenciales para el logro de los fines de la educación, deben recuperarse. Más allá de su tarea en la preparación académica de sus alumnos, son ejemplo y guía para la educación en valores y en derechos humanos.

Es preciso que los valores se enseñen desde la familia y se afiancen en el estudiantado con el apoyo del personal docente en turno, de tal manera que en el transcurso de sus unidades de aprendizaje tengan presente los valores en su nivel de educación y correlacionarlo con su vida personal y el mundo laboral. Es conveniente 
que la relación de la práctica docente y la educación en valores se cumpla en todos los aspectos de la vida, para ello es preciso evitar la crisis valoral que se ha convertido en una problemática a nivel país, cuestión que ha llevado a efectuar la presente revisión sistemática de literatura, a fin de conocer las investigaciones desde el estudio de los valores en la educación superior ejecutadas en los últimos años y las aportaciones desde el punto de vista de los autores seleccionados para el estado del arte en cuestión.

\section{MARCo TEÓRICo}

\section{La educación en valores en el ámbito universitario}

En la familia se encuentran los principios de los comportamientos, y se establecen los primeros valores que el individuo adoptará para regir su vida, articulándolos con la escuela el individuo se desenvolverá humanamente, desarrollando así actitudes y conductas que le permitirán ejercer valores hacia un beneficio personal y social por medio de la educación; como lo menciona Herrera (2007) quien refiere que es importante destacar y no se debe olvidar que la escuela tanto como la familia, de forma coordinada y corresponsable, tienen un propósito netamente educativo y educar consiste fundamentalmente en desarrollar hábitos y, formar educativamente hábitos significa, procurar actitudes que conduzcan a la formación y práctica de valores, dirigidos hacia el bien (virtudes).

El ser humano se desarrolla en relación con el contexto en el que vive la familia, esto condiciona los procesos de su personalidad y de la adaptación en el círculo social en el que se desenvuelva en tanto que puede tener respuestas de comportamiento negativas o positivas. Citando a Quintana (1993) quien define a la familia como una institución educativa, pero cuyos miembros no han recibido una preparación específica para esta función. La familia tiene a su cargo situaciones difíciles de resolver en la educación como el fomento de buenos modales formación de comportamientos y actitudes ante ciertas situaciones, ideales y sobre todo trasmisión de valores.

Definir la educación en valores en el contexto de la Educación Superior, no es tarea fácil, se requiere considerar un constructo teórico donde confluyen diversos aspectos y elementos que la integran como a continuación se presenta.

\section{Educación}

La educación ha formado a la sociedad según la cultura a la que pertenece en donde existen hábitos, costumbres e ideologías particulares; sin embargo el objetivo de la educación no se centra en desarrollar talento intelectual; sino de dotar al ser humano de capacidades relacionadas con la humanización, donde los valores, la ética, la moral, las normas de convivencia y la parte afectiva se desenvuelvan en su máximo esplendor, en palabras de García, Ruiz, y García (2011) la educación es el proceso de convertirse en persona es decir, aquella acción gradual y permanente en el tiempo dirigida al logro de la plena humanización, entendiéndose ésta tanto en una dimensión individual, como social propia de todo ser humano. En esta misma línea, se concibe educación como todo proceso consolidado y dirigido a la optimización de la persona en el ser, el conocer, el hacer y el convivir. Como se puede señalar, la educación también tiene lugar para formar al ser humano integralmente para que este actúe de manera libre y soberanamente y demostrando en cada comportamiento los valores que lo rigen. 


\section{Valores}

Para concebir qué es la educación en valores es necesario reconocer que la educación ya es un valor, considerar los valores como características morales de manera positiva, se estima una fuerte influencia en las actitudes, convirtiéndose en la base de una sana convivencia social y personal donde el individuo demuestra sus principios y virtudes a través de ciertas circunstancias, para Penas (2008) los valores son características de la acción humana. Desde la perspectiva moral, son el conjunto de normas empleadas para llevar a cabo una actividad o juicio. Los valores son intrínsecos a la acción humana y mueven la conducta, orientan la vida y marcan la personalidad. La vida de cada quien se va a definir en función de los valores que se elijan. A través de los valores se puede alcanzar el equilibrio y armonía entre el individuo y su contexto y así logra regir su propia vida e impactar a través de la promoción de éstos hacía la sociedad y la familia como primer núcleo de contacto social.

\section{Educación en valores}

Retomando que a través de la educación el individuo puede lograr la humanización por medio de un proceso para convertirse en una persona de bien con actitudes reflexivas conforme a sus principios donde ejerza libertad y autonomía al momento de tomar decisiones las cuales serán respaldadas por la jerarquía de valores que le rijan, es entonces que la educación en valores se traduce en un proceso humanizador que marca al individuo en el transcurso de su vida, lo cual permitirá las buenas relaciones a nivel personal y social manifestando las virtudes que le distinguen. Desde la perspectiva de Merma, Peiró i Gregòri, y Gavilán (2013), la educación en valores es el proceso mediante el cual se guía y se forma a los alumnos para que sean capaces de utilizar su experiencia axiológica de manera consciente para construir su proyecto personal de vida. Su objetivo es el desarrollo de destrezas, hábitos, actitudes y conocimientos, que capaciten a las personas para hacer, intervenir, comportarse, aprender e interrelacionarse con los demás, con base en valores. En este sentido, la educación en valores tendrá que proporcionar al estudiante un desarrollo integral, por medio de una escala de valores relacionada a las necesidades del contexto social, profesional y cultural actual, ello le permitirá ejercer con plena responsabilidad y conciencia su profesión, alcanzando de esta manera una vida plena y de ejemplo a seguir por los que le rodean.

En cambio, Latapí (2001) define la educación en valores como el esfuerzo sistemático por ayudar a los educandos a adquirir aquellas cualidades de su personalidad que se consideran deseables en los diversos ámbitos de su desarrollo humano, y particularmente aquellas que se relacionan con el uso responsable de su libertad. Entonces queda claro lo que Martínez (2006) asegura al establecer que una formación universitaria de calidad no puede separar la formación profesional de la formación ciudadana y que, por tanto, la universidad debe ser un espacio de construcción de valores.

\section{Docentes universitarios}

La educación en valores tiene un impacto a través de la intervención del personal docente universitario, porque es por medio de su práctica que ejerce sus propios valores de acuerdo con las características que lo distinguen de su propia personalidad y carácter. Cada docente debe ser consciente de que el alumnado se encuentra en una etapa de construcción como profesionistas y como personas por lo que fundan sus propios valores a través de lo que observan y aprenden de sus docentes como su principal referente; por ello es importante destacar que durante su práctica no solo transmite conocimientos científicos y/o tecnológicos sino que también es un ejemplo y una guía que además de instruir impacta la vida del estudiantado a través 
de sus comportamientos, actitudes, forma de resolver situaciones y enfrentar problemas; entonces Estrada (2012) menciona:

Es por esto que los profesores deben enseñar los modos de conducta, no exigir una que no ha sido previamente enseñada; proporcionar medios que sirvan de apoyo a las conductas aprendidas; conocer y satisfacer las necesidades de los estudiantes y crear nuevas necesidades; estimular las buenas conductas; mantener las exigencias, garantizar que el alumno conozca si posee una conducta correcta o no, y que aprenda a analizar los errores cometidos y cómo arreglarlos (p. 263).

En este sentido, es inexcusable que las IES replanten acciones de manera integral, considerando en ello estudios del micro y macro ambiente educativo donde se identifiquen fuerzas favorables y áreas de oportunidad relacionadas con valores en la educación, con la firme intención de proponer soluciones a través de la formación profesional del y la docente, quien es el principal constructor de valores en los educandos en el proceso de enseñanza aprendizaje.

Como se ha señalado, el profesorado debe estar preparado no solo en conocimientos y habilidades sino en ser transformado a través de la profesionalización integral que le permita asumir el desafío por una enseñanza en la formación de valores hacia los estudiantes; quienes a través de la educación valoral se aprovisionaran de las herramientas necesarias para desarrollarse eficientemente en un entorno económico y social complejo.

\section{Educación Superior}

A través de políticas públicas influenciadas por un mundo globalizado donde las economías abiertas han generado el estado de la competitividad por conseguir posiciones privilegiadas en los mercados nacionales e internacionales, los Modelos Educativos en las Instituciones de Nivel Superior se han visto repercutidos para generar las condiciones de eficiencia y eficacia de un mercado laboral. Es por eso que Cantú (2018) indica que indiscutiblemente, el escenario universitario es el lugar, por excelencia, donde se crean los espacios para dar respuesta a las problemáticas actuales y a los nuevos retos sociales en la nación. Si bien es cierto, en la actualidad se han considerado estos aspectos que han llevado a las IES a preocuparse por diseñar planes y programas de estudio enfocados a las demandas de una economía globalizada.

Según Chapa y Martínez (2015), notifican que las IES tienen la tarea de contribuir a que los futuros profesionales desarrollen una visión y sentido ético, que pueda orientar a la práctica y que refleje en sus tareas a los valores en un conjunto: responsabilidad, solidaridad, sentido de la justicia, servicio a otros. Las Instituciones de Enseñanza Superior tendrán que reorientar sus funciones en vista de una transformación de la educación.

Esta investigación tiene por objetivo abordar e identificar la producción académica en la temática de educación en valores para la Educación Superior a través de la metodología de revisión sistemática de literatura cualitativa, de las investigaciones empíricas desarrolladas con cohorte 2013-2018, en el contexto de Latinoamérica, para ello es pertinente identificar las teorías más representativas que son empleadas en las investigaciones relacionadas con la educación en valores, así mismo conocer las variables y los actores sociales que cada investigador establece para proponer escala de valores.

\section{Metodología}

En esta investigación se empleó la metodología de revisión sistemática de literatura, la cual tiene como propósito integrar de forma objetiva y sistemática los resultados de los estudios empíricos sobre un determinado problema de investigación, con objeto de determinar el 'estado del arte' en ese campo de estudio (Sánchez-Meca, 2010).

Para esta investigación sistemática, se empleó el corte cualitativo a partir de un estudio integrativo, observacional, retrospectivo, secundario en el cual se combinan estudios que examinan la misma pregunta. En 
este tipo de investigación se requiere definir una pregunta claramente, especificar los criterios de inclusión y exclusión de los estudios, formulación del plan de búsqueda de la literatura, registro de los datos y evaluación de la calidad de los estudios seleccionados e interpretación y presentación de los resultados (Beltrán, 2005).

A continuación, se describen los criterios empleados para la revisión sistemática para Latinoamérica:

a) Preguntas de investigación:

Q.1.- ¿Cuáles son las teorías representativas que emplean los autores en el campo de la educación en valores?

Q.2.- ¿Cuáles son las variables que se relacionan en el ámbito de la educación en valores desde la perspectiva de los diferentes autores?

Q.3.- ¿Cuáles son los actores sociales involucrados que cada autor propone para constituir una escala de valores?

b) Criterios de inclusión y exclusión:

Los criterios de inclusión se muestran a continuación:

1. Búsqueda de estudios en bases de datos en Dialnet, Redalyc y Scielo,

2. Estudios pertenecientes al campo de la educación en valores en el nivel superior,

3. Investigaciones desarrolladas en el periodo 2013-2018,

4. Investigaciones de acceso abierto.

c) Formulación del plan de búsqueda de la literatura y registro de datos

La búsqueda se efectuó a partir del mes de octubre y noviembre de 2018. Se consultaron bases de datos antes mencionadas, donde se indagaron 35 artículos de investigación donde la selección de los artículos fue con base en los criterios de inclusión, estableciendo un total de 14 artículos, de los cuales se analizó en dos secciones; la parte contextual y de contenido. El registro de datos se muestra a continuación, donde se mencionan los elementos contemplados:

- Para el contexto se incluyeron aspectos como: autor y año de la publicación; país; palabras clave; enfoque metodológico; hallazgos y limitantes (ver Tabla 1).

- Para el contenido se incluyeron aspectos relacionados con la educación en valores como: autor y año de la publicación; teorías representativas (Socioeducativa, Cognitiva, Constructivista, Pedagogía Social, Socio-Humanista y Psicopedagógica), variables inmersas en el ámbito de la educación en valores (Política Educativa, Política Institucional, Docentes, Currículum, Familia, Pedagogía, Sociedad, Cultura, Ética y Moral). Por último, se consideraron los actores involucrados para el diseño de una escala de valores que cada autor presentó (ver Tablas 2 y 3 ).

d) Evaluación de la calidad de los estudios seleccionados

El análisis se ejecutó, de acuerdo con el principio de la intención a tratar referente al estado del arte de las investigaciones recientes (2013-2018) en los artículos que estudian los aspectos académicos relacionados con la educación, el análisis se cumplió con base en criterios de búsqueda los cuales estaban compuestos por palabras clave: Universidad, Valores, Educación, Docentes y Estudiantes (ver Tabla 1). 
TABLA 1.

Análisis de los principales artículos publicados de Latinoamérica sobre la Educación en Valores para el Nivel Superior.

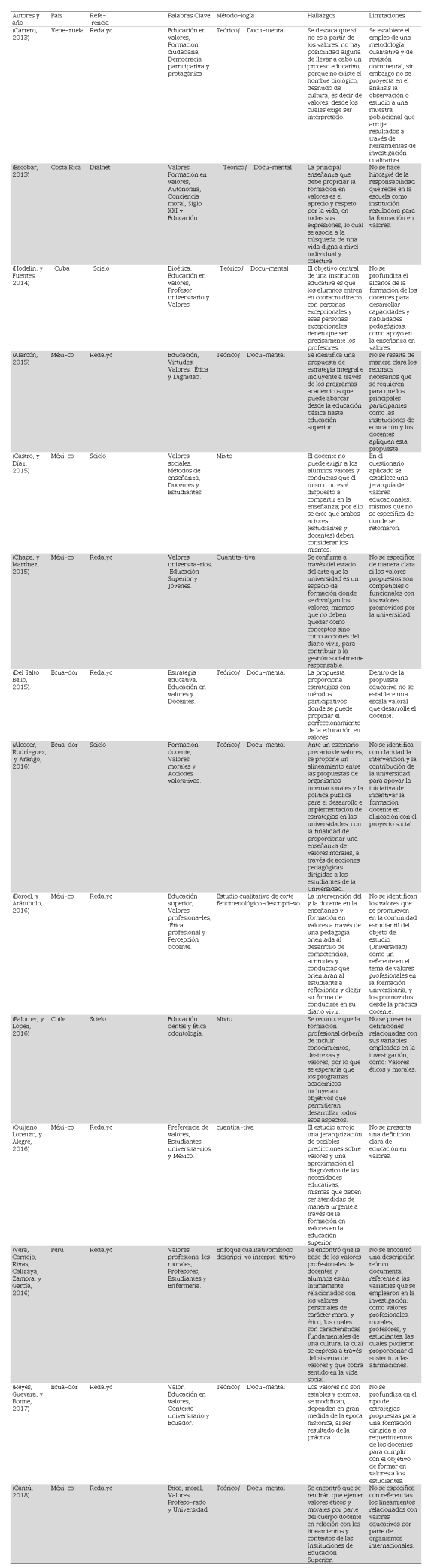


Fuente: Elaboración propia.

\section{Resultados}

De los catorce artículos analizados, seis se llevaron a cabo en México, tres en Ecuador, uno en Costa Rica, uno en Chile, uno en Cuba, uno en Perú y uno en Venezuela (ver Figura 1). Nueve de ellos residían de bases de datos en Redalyc, cuatro en Scielo y uno en Dialnet, los resultados e interpretación se muestran a continuación:

- Las palabras clave, representadas y argumentadas a través de los principales conceptos observados en las investigaciones fueron: Universidad, Docentes, Estudiantes, Formación en Valores, Ética, Moral, Enseñanza y Profesionales.

- En el enfoque metodológico e instrumentos empleados se identificó que en ocho artículos su enfoque era Teórico Documental, dos Cualitativos, dos Cuantitativo y dos Mixtos. Los instrumentos más empleados fueron: la entrevista y el cuestionario, la observación fue escasa.

- Los principales hallazgos reconocidos en las investigaciones son seis factores importantes, los cuales influyen en la educación en valores para el nivel superior, como: 1. El impacto para que las IES replanten su propósito y compromiso con la sociedad, para que a través de la instrucción se logre una formación integral y una humanización, 2. La capacitación al cuerpo docente para concientizar que a través del ejemplo se puede conseguir una educación con valores éticos y morales porque no se puede exigir algo que no se da, 3. La importancia de contar con una escala de valores que conduzcan a los estudiantes a enfrentar los retos y desafíos de un contexto social, económico, político y cultural desafiante, 4. La necesidad de emplear una pedagogía orientada al desarrollo de competencias, actitudes y conductas que orienten al estudiante a tomar decisiones de manera analítica y reflexiva y con ello elegir su forma de conducirse en su diario vivir, 5. El impacto en beneficio de la sociedad al implementar a través de políticas educativas la educación en valores lo que repercutirá en el crecimiento y desarrollo de una mejor sociedad, y 6. A través de la implementación de metodologías relacionadas con el aprendizaje-servicio, se puede lograr la practicidad de valores en estudiantes y docentes por medio de la vinculación universidad-Programas Sociales donde los alumnos y maestros pongan al servicio de la sociedad sus valores y conocimientos; así el estudiantado vivirá en otros ambientes la reflexión y análisis que trae consigo el conocerse a sí mismos.

- Las limitantes encontradas hacen referencia en que no se ha definido con claridad las variables inmersas en el ámbito de la educación en valores, a través de la fundamentación de un marco teórico sólido. 


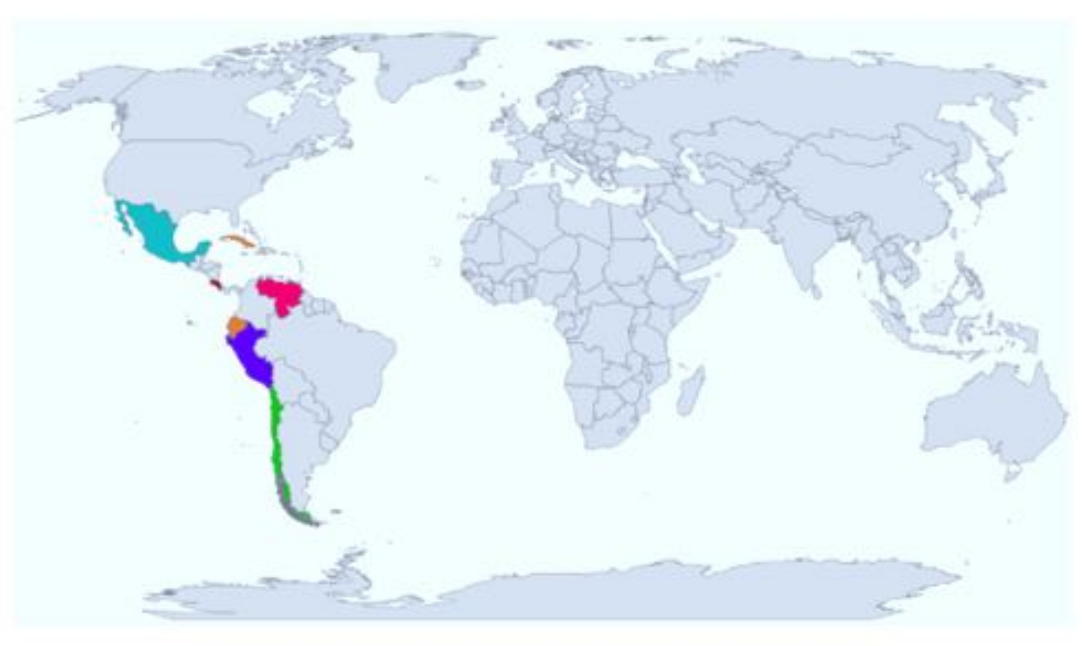

FIGURA 1

Representación de los 14 estudios en países analizados para el tema de educación en valores Fuente: Elaboración propia.

De acuerdo con el análisis e interpretación de los datos, y teniendo como referente las preguntas de investigación se examinaron aspectos relacionados con la educación en valores, dividida en tres secciones y se encontró lo siguiente:

Q.1.- Primera Sección. Las teorías representativas que emplearon los autores para explicar la realidad en el campo de la educación son cinco; estas son aplicables a la educación; cabe destacar que a través del estado del arte se identificó que solo nueve investigaciones fueron sustentadas a través de teorías representativas en la educación; entre ellas se encuentran: 1. Carrero (2013) con las teorías Socio-educativa y Constructivista; 2. Escobar (2013) con la teoría Constructivista; 3. Hodelín y Fuentes (2014) con las teorías Constructivista y Socio-Humanista; 4. Alarcón (2015) con la teoría Socio-Humanista; 5. Alcocer et al. (2016) con la teoría Constructivista; 6. Boroel y Arámbulo (2016) con las teorías Socio-Humanista y Psico-Pedagógica; 7. Vera et al. (2016) con la teoría Socio-Humanista; 8. Reyes, Guevara y Bonne (2017) con la teoría Pedagogía Social y por último 9. Cantú-Martínez (2018) con la teoría Pedagogía Social. Los autores Castro y Díaz (2015); Chapa y Martínez (2015); Del Salto Bello (2015); Palomer y López (2016) así como Quijano, Lorenzo y Alegre (2016) no emplearon en sus investigaciones un enfoque teórico donde se explique de manera sustentada los aspectos que convergen en el estudio de los valores en la educación superior desde una perspectiva concreta, es decir dando por hecho una serie de axiomas a partir de los cuales se elaboraron estas investigaciones para seguir produciendo conocimiento científico. Cabe destacar que la teoría que no se identificó en ninguno de los estudios es la Cognitiva (ver Tabla 2). 
TABLA 2

Teorías representativas que emplearon los autores en el campo de la Educación en Valores para el Nivel Superior.

\begin{tabular}{lllllll}
\hline Autores y año & $\begin{array}{l}\text { Socio- } \\
\text { educa- } \\
\text { tiva }\end{array}$ & $\begin{array}{l}\text { Cogni- } \\
\text { tiva }\end{array}$ & $\begin{array}{l}\text { Constru- } \\
\text { ctivista }\end{array}$ & $\begin{array}{l}\text { Pedago- } \\
\text { gia Social }\end{array}$ & $\begin{array}{l}\text { Socio- } \\
\text { Huma- } \\
\text { nista }\end{array}$ & $\begin{array}{l}\text { Psico- } \\
\text { pedagó- } \\
\text { gica }\end{array}$ \\
\hline (Carrero, 2013) & $\checkmark$ & $\mathrm{X}$ & $\checkmark$ & $\mathrm{X}$ & $\mathrm{X}$ & $\mathrm{X}$ \\
$\begin{array}{l}\text { (Escobar, 2013 } \\
\text { (Hodelín, y Fuentes, }\end{array}$ & $\mathrm{X}$ & $\mathrm{X}$ & $\checkmark$ & $\mathrm{X}$ & $\mathrm{X}$ & $\mathrm{X}$ \\
2014) & $\mathrm{X}$ & $\checkmark$ & $\mathrm{X}$ & $\checkmark$ & $\mathrm{X}$ \\
(Alarcón, 2015) & $\mathrm{X}$ & $\mathrm{X}$ & $\mathrm{X}$ & $\mathrm{X}$ & $\checkmark$ & $\mathrm{X}$ \\
(Castro, y Díaz, 2015) & $\mathrm{X}$ & $\mathrm{X}$ & $\mathrm{X}$ & $\mathrm{X}$ & $\mathrm{X}$ & $\mathrm{X}$ \\
(Chapa, y Martínez, & $\mathrm{X}$ & $\mathrm{X}$ & $\mathrm{X}$ & $\mathrm{X}$ & $\mathrm{X}$ & $\mathrm{X}$ \\
2015) & & & & & & \\
(Del Salto Bello, 2015) & $\mathrm{X}$ & $\mathrm{X}$ & $\mathrm{X}$ & $\mathrm{X}$ & $\mathrm{X}$ & $\mathrm{X}$ \\
(Alcocer et al., 2016) & $\mathrm{X}$ & $\mathrm{X}$ & $\checkmark$ & $\mathrm{X}$ & $\mathrm{X}$ & $\mathrm{X}$ \\
$\begin{array}{l}\text { (Boroel, y Arámbulo, } \\
\text { 2016) }\end{array}$ & $\mathrm{X}$ & $\mathrm{X}$ & $\mathrm{X}$ & $\mathrm{X}$ & $\checkmark$ & $\checkmark$ \\
(Palomer, y López, 2016) & $\mathrm{X}$ & $\mathrm{X}$ & $\mathrm{X}$ & $\mathrm{X}$ & $\mathrm{X}$ & $\mathrm{X}$ \\
$\begin{array}{l}\text { (Quijano et al., 2016) } \\
\text { (Vera et al., 2016) }\end{array}$ & $\mathrm{X}$ & $\mathrm{X}$ & $\mathrm{X}$ & $\mathrm{X}$ & $\mathrm{X}$ & $\mathrm{X}$ \\
(Reyes et al., 2017) & $\mathrm{X}$ & $\mathrm{X}$ & $\mathrm{X}$ & $\mathrm{X}$ & $\checkmark$ & $\mathrm{X}$ \\
(Cantú, 2018) & $\mathrm{X}$ & $\mathrm{X}$ & $\mathrm{X}$ & $\checkmark$ & $\mathrm{X}$ & $\mathrm{X}$ \\
\hline & & & & & & $\mathrm{X}$ \\
\hline
\end{tabular}

Fuente: Elaboración propia.

Q.2.- Segunda Sección. Una vez analizadas las investigaciones, se tuvo como resultado que las variables que los autores emplearon y que se relacionan en el ámbito de la educación en valores se mencionan en un rango de mayor a menor empleo en las exploraciones como son: Sociedad, Ética y Moral por doce investigaciones; Docentes por nueve artículos; Política Institucional por ocho estudios; Currículum por seis trabajos académicos; así también Política Educativa y Cultura por cinco autores; Pedagogía por tres diferentes indagaciones, y por último Familia por solo dos revisiones. Las investigaciones que emplearon hasta menos de tres variables son: Escobar (2013), Alarcón (2015) y también Chapa y Martínez (2015) con dos variables; Castro y Díaz (2015) y Reyes et al. (2017) con tres variables (ver Tabla 3). 
TABLA 3

Variables que se relacionan con la Educación en Valores para el Nivel Superior desde la perspectiva de diversos autores.

\begin{tabular}{|c|c|c|c|c|c|c|c|c|c|}
\hline Autores y año & $\begin{array}{l}\text { Política } \\
\text { Educa- } \\
\text { tiva }\end{array}$ & $\begin{array}{l}\text { Política } \\
\text { Institu- } \\
\text { Cional }\end{array}$ & $\begin{array}{l}\text { Docen- } \\
\text { tes }\end{array}$ & $\begin{array}{l}\text { Curri- } \\
\text { Culum }\end{array}$ & $\begin{array}{l}\text { Fami- } \\
\text { lia }\end{array}$ & $\begin{array}{l}\text { Pedago- } \\
\text { gía }\end{array}$ & $\begin{array}{l}\text { Socie- } \\
\text { dad }\end{array}$ & $\begin{array}{l}\text { Cultu- } \\
\text { ra }\end{array}$ & $\begin{array}{l}\text { Ética } \\
\text { y } \\
\text { Moral }\end{array}$ \\
\hline (Carrero, 2013) & $r$ & $\mathrm{X}$ & $\mathrm{X}$ & $r$ & $\mathrm{X}$ & $\mathrm{X}$ & $r$ & $r$ & $r$ \\
\hline (Escobar, 2013) & $\mathrm{X}$ & $\mathrm{X}$ & $\mathrm{X}$ & $\mathrm{X}$ & $\mathrm{X}$ & $r$ & $\checkmark$ & $\mathrm{X}$ & $\mathrm{X}$ \\
\hline $\begin{array}{l}\text { (Hodelín, y } \\
\text { Fuentes, 2014) }\end{array}$ & $\checkmark$ & $\checkmark$ & $\checkmark$ & $r$ & $\mathrm{X}$ & $\mathrm{X}$ & $r$ & $r$ & $r$ \\
\hline $\begin{array}{l}\text { (Alarcón, } \\
\text { 2015) }\end{array}$ & $\mathrm{X}$ & $\mathrm{X}$ & $\mathrm{X}$ & $\mathrm{X}$ & $\mathrm{X}$ & $\mathrm{X}$ & $r$ & $\mathrm{X}$ & $r$ \\
\hline $\begin{array}{l}\text { (Castro, y Díaz, } \\
\text { 2015) }\end{array}$ & $\mathrm{X}$ & $\mathrm{X}$ & $\checkmark$ & $r$ & $\mathrm{X}$ & $\mathrm{X}$ & $\mathrm{X}$ & $\mathrm{X}$ & $r$ \\
\hline $\begin{array}{l}\text { (Chapa, y } \\
\text { Martínez, } \\
\text { 2015) }\end{array}$ & $\mathrm{X}$ & $\checkmark$ & $\mathrm{X}$ & $\mathrm{X}$ & $\mathrm{X}$ & $\mathrm{X}$ & $\mathrm{X}$ & $\mathrm{X}$ & $r$ \\
\hline $\begin{array}{l}\text { (Del Salto Bello, } \\
\text { 2015) }\end{array}$ & $\checkmark$ & $\checkmark$ & $\checkmark$ & $\mathrm{x}$ & $\mathrm{X}$ & $\checkmark$ & $r$ & $r$ & $r$ \\
\hline $\begin{array}{l}\text { (Alcocer et al., } \\
\text { 2016) }\end{array}$ & $\checkmark$ & $\checkmark$ & $\checkmark$ & $\mathrm{X}$ & $\mathrm{X}$ & $\mathrm{X}$ & $r$ & $\mathrm{X}$ & $\checkmark$ \\
\hline $\begin{array}{l}\text { (Boroel, y } \\
\text { Arámbulo, } \\
2016 \text { ) }\end{array}$ & $\mathrm{X}$ & $\checkmark$ & $\checkmark$ & $\checkmark$ & $\checkmark$ & $\checkmark$ & $r$ & $r$ & $\checkmark$ \\
\hline $\begin{array}{l}\text { (Palomer, y } \\
\text { López, 2016) }\end{array}$ & $\mathrm{X}$ & $\checkmark$ & $\checkmark$ & $\checkmark$ & $\mathrm{X}$ & $\mathrm{X}$ & $\checkmark$ & $\mathrm{X}$ & $\checkmark$ \\
\hline $\begin{array}{l}\text { Quijano et al., } \\
2016 \text { ) }\end{array}$ & $\mathrm{X}$ & $\checkmark$ & $\mathrm{X}$ & $\checkmark$ & $\mathrm{X}$ & $\mathrm{X}$ & $r$ & $\mathrm{X}$ & $r$ \\
\hline $\begin{array}{l}\text { (Vera et al., } \\
2016)\end{array}$ & $\mathrm{X}$ & $\mathrm{X}$ & $\checkmark$ & $\mathrm{X}$ & $\mathrm{X}$ & $\mathrm{X}$ & $\checkmark$ & $\checkmark$ & $\checkmark$ \\
\hline $\begin{array}{l}\text { (Reyes et al., } \\
2017)\end{array}$ & $\mathrm{X}$ & $\mathrm{X}$ & $\checkmark$ & $\mathrm{X}$ & $r$ & $\mathrm{x}$ & $r$ & $\mathrm{X}$ & $\mathrm{X}$ \\
\hline (Cantú, 2018) & $r$ & $r$ & $r$ & $\mathrm{X}$ & $\mathrm{X}$ & $\mathrm{X}$ & $r$ & $\mathrm{X}$ & $\checkmark$ \\
\hline
\end{tabular}

Fuente: Elaboración propia

Q.3.- Tercera Sección. Los autores plantearon cinco actores sociales involucrados en la educación en valores; estos hacen referencia a diversas escalas valorales como a continuación se presenta (ver Tabla 4).

- El primer actor: se enfoca a los estudiantes como Carrero (2013); Escobar (2013); Hodelín y Fuentes (2014); Palomer y López (2016) y Quijano et al. (2016) refieren al exponer a través de sus estudios una propuesta de valores dirigida a estudiantado universitario, dado que serán los futuros profesionistas que se enfrentarán a retos en diversos contextos como en el profesional, social y cultural, por ello se requiere que se encuentren preparados con valores sólidos y pertinentes lo cual les llevarán a tomar mejores decisiones y así contribuirán a un mejor estilo de vida.

- Los segundos actores: docentes y estudiantes como lo refieren Castro y Díaz (2015); Boroel y Arámburo (2016) y Vera et al. (2016) al proponer una escala valoral dirigida para ambos actores donde se establece que los docentes no pueden demandar conductas y actitudes a los estudiantes sin antes ser ellos los primeros en fomentarlos a través de su propia personalidad así como de las estrategias de enseñanza aprendizaje que empleen en el salón de clases, lo cual aporte para una educación en valores que les brindara a los estudiantes las herramientas necesarias para enfrentar la vida, es decir; el docente exige a sus alumnos de acuerdo a lo que el primero está dispuesto a dar.

- En el tercer actor involucrado: se encuentran los docentes, derivado de las investigaciones de Reyes et al. (2017) y Cantú (2018) quienes hacen referencian en sus investigaciones que las universidades deben contemplar docentes capacitados, comprometidos y consientes de ser ejemplo a través de comportamientos y actitudes que influyen a través del ejercicio y la práctica de hábitos que 
demuestren valores éticos y morales. Así mismo, consideran que el docente es el principal responsable de la transmisión de valores, y con ello proponen una escala valoral hacia el docente.

- Dentro del cuarto actor: se encuentran las investigaciones centradas en Alarcón (2015) quien propone una estrategia integral a través de una escala valoral dirigida desde el nivel básico hasta el nivel superior, haciendo referencia de incluir en la educación virtudes y valores que permita el desarrollo y formación del carácter del educando; así mismo se involucra la educación en valores a través de Alcocer et al. (2016) donde se distingue en señalar que los sistemas educativos deben instaurar una escala valoral con base en un alineamiento de propuestas por los organismos internacionales con la política pública educativa, con la intención de propiciar una educación en valores por medio de acciones pedagógicas considerando en ello la propuesta valoral que los autores establecen en su investigación.

- Para el quinto actor: se localiza la Universidad en el estudio elaborado por los autores Chapa y Martínez (2015) se destaca la necesidad de establecer una escala valoral dirigidas a las IES; por ser espacios de formación donde se divulgan valores y se reconoce que a través de la institución se debe formar al ciudadano. Se resalta que el autor Del Salto Bello (2015) dirige su investigación hacia los docentes; sin embargo, no propone clasificación de valores en su estudio. Afirma que la educación en valores debe propiciar el aprecio y el respeto por la vida.

TABLA 4

Actores sociales involucrados para el diseño de una escala de valores para el Nivel Superior

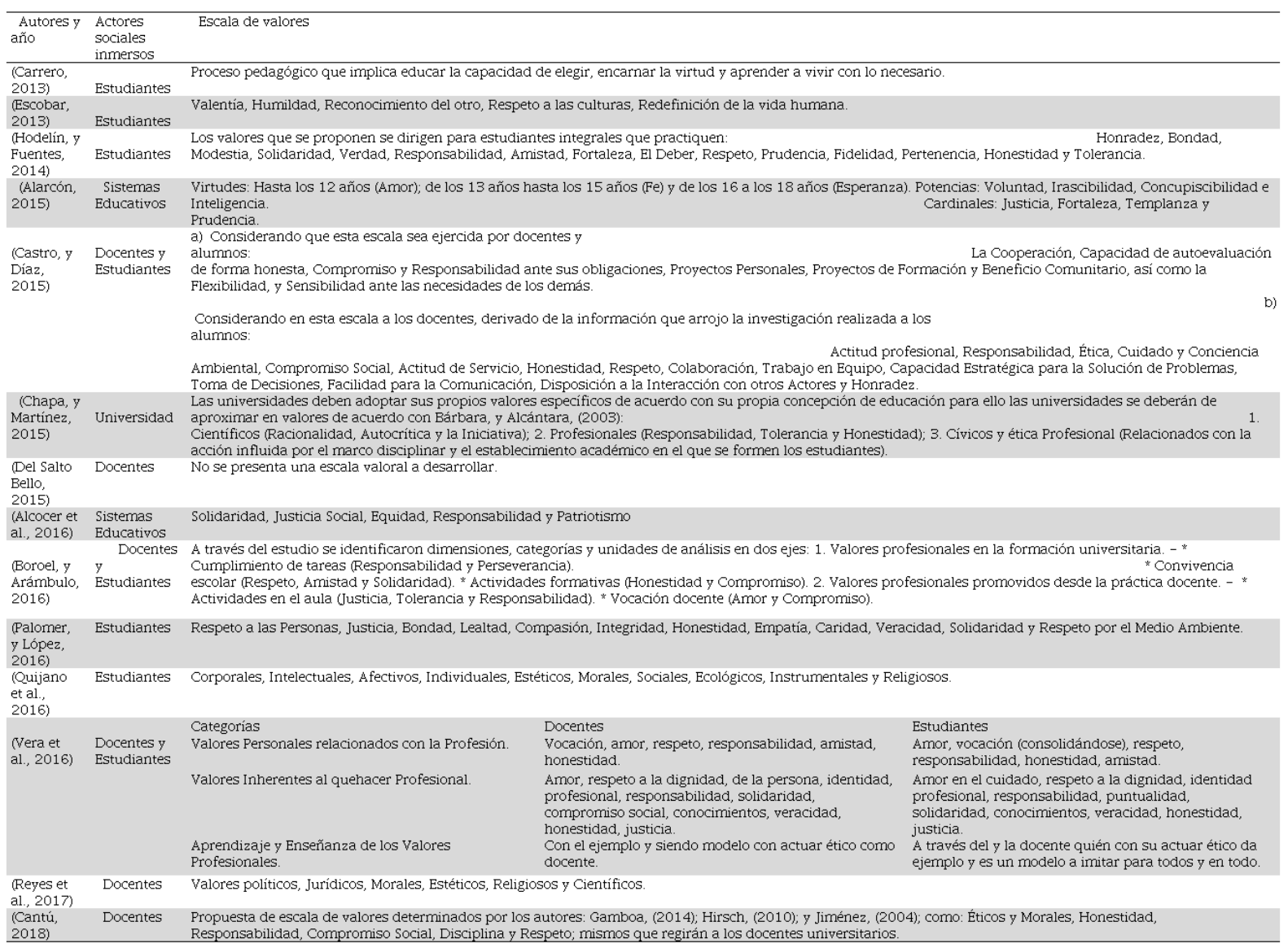

Fuente: Elaboración propia, con base en el estado del arte en cuestión. 


\section{Discusiones}

El objetivo que direccionó esta investigación para Latinoamérica fue desarrollar un análisis desde la perspectiva de distintos autores para obtener a través del estado del arte una revisión sistemática de literatura (SLR) de investigaciones recientes en artículos que estudian los aspectos académicos relacionados con la educación en valores en el nivel superior, los resultados obtenidos han aportado información acerca de la situación actual de la educación en valores para el nivel superior.

De acuerdo con los planteamientos por los autores analizados queda de manifiesto la prioridad de que la educación superior refrende su compromiso ante la sociedad al comprometerse con el egreso de profesionistas con un desarrollo integral y humano quienes forjarán una personalidad de pensamiento crítico y reflexivo, porque es a través de la preparación integral fundamentada en valores éticos y morales que se responde a la necesidad de enfrentar las dificultades reales de una sociedad, como lo proponen los autores Alcocer et al. (2016) quienes afirman que las instituciones superiores deben ir más allá de formar profesionales, para formar ciudadanos comprometidos, activos, humanistas, abiertos al debate de ideas, cimentados en valores orientados al bien común, a formar ciudadanos que participen activamente en la sociedad.

Así mismo, es pertinente reconocer que la revisión arrojó datos sobre el estado del conocimiento al destacar propuestas de escalas valorales hacía los actores sociales implicados en la educación como son: los sistemas educativos para todos los niveles de educación, en especial para el Nivel Superior Universitario y en específico para sus estudiantes y docentes. Lo que indica como reto para futuras investigaciones por autores interesados en el tema de educación en valores para la educación superior; la publicación de resultados de la implementación y puesta en marcha de las propuestas valorales que se han abordado e identificado en este estudio por autores de Latinoamérica; quienes plantean y sitúan como válidas y pertinentes para el contexto referente a las necesidades sociales, económicas, políticas y culturales.

Si bien las investigaciones analizadas no cubren el total de los artículos verificados en el contexto de Latinoamérica, a pesar de ello arrojaron algunos indicadores sobre el estado del conocimiento del tema como a continuación se menciona:

En las investigaciones se pudo constatar que hubo un predominio en el enfoque Teórico/Documental el cual es congruente con el abordaje epistemológico de educación en valores, sin embargo, poco recurrente en analizar la influencia que la familia representa en la educación en valores como primera institución social formadora donde se desenvuelve el ser humano, sin embargo para este tipo de investigación, sus variables recurrentes en el ámbito de la educación fueron la sociedad, porque se reconoce la necesidad de constituir y formar al ser humano por valores éticos y morales que serán expuestos en correlación y atención y a las necesidades del tejido social. Los países impulsores de las investigaciones bajo esta premisa fueron: Ecuador con tres, México con dos, Costa Rica, Cuba y por último Venezuela con solo una investigación.

Posteriormente hubo preponderancia en pesquisas relacionadas con estudios de carácter cualitativo, que revelaron la relación de estudios de valores enfocados a estudiantes y docentes, donde se resaltó la participación del y la docente como un agente de cambio quien debe someterse a una formación para que a través de su profesión contribuya en una enseñanza donde manifieste valores éticos y morales a los estudiantes con relación con el profesional que requiere la sociedad; contribuyendo entonces de esta manera a lo que proponen Hodelín y Fuentes (2014) cuando mencionan que el alumnado también debe construir sus propios valores, en referencia a los que los docentes enseñan, reflejan y promueven en el proceso de enseñanzaaprendizaje. En los estudios de este corte figuran México y Perú con una investigación por cada país.

En seguida, para los estudios de tipo cuantitativo se constató que a través de las investigaciones que se analizaron, éstas se enfocaron a medir la elección y prioridad de valores educativos en los estudiantes, lo que evidencia que existen posibles predicciones sobre los valores que demuestran los estudiantes; en este sentido le corresponde atender a las instituciones de nivel superior la factibilidad de dirigir y orientar a través de una 
propuesta de escalafón valoral que atienda las necesidades educativas de desarrollo integral en los estudiantes. Las investigaciones analizadas en este tipo de estudio fueron dos de México.

Por último, se examinaron dos artículos de corte mixto donde se verificaron a través de la medición de valores en estudiantes con la participación de la observación por docentes, la sobresaliente necesidad de que las instituciones de nivel superior deban concentrarse en los planes y programas de estudio en el que se destaquen objetivos encaminados a desarrollar aspectos relacionados con valores éticos y morales, como lo destacan los autores Castro y Díaz (2015) así como Palomer y López (2016) al reconocer que las Instituciones de Educación Superior deben preparar individuos con conocimientos amplios para el abordaje de los problemas humanos de su entorno, y esto sólo se logra si en el diseño curricular se imparten los valores conjuntamente con los conocimientos de la ciencia. Los artículos para esta investigación son de México y Chile.

\section{Conclusiones}

En conclusión en Latinoamérica, es necesario reconocer que, para lograr una implementación de una educación en valores para el nivel superior, se necesita de la participación integral de los diversos actores sociales implícitos en la educación, a través del diseño de políticas educativas en valores; dado que en la actualidad no se han hecho manifiestas escalas valorales las cuales direccionen la educación; así mismo de la intervención de las IES quienes deben de garantizar la formación integral y humana de profesionistas que se desenvuelven en diversos ámbitos sociales y económicos del contexto del siglo XXI, tal y como lo aseveran Chapa y Martínez (2015) a la Universidad como una importante vía para la formación de las nuevas generaciones, deben promover los valores en la ejecución de la gestión socialmente responsable de la formación integral, aplicación del conocimiento y la cultura, y del funcionamiento institucional en todos sus ámbitos, considerando en ello lo que Carrero (2013) menciona en relación a que la escuela recibe el cargo de educar a los alumnos para los valores y también el de preparar al estudiante para la vida. Se hace vital y necesario continuar con investigaciones aplicadas al ámbito de la educación en valores en el nivel superior.

\section{REFERENCIAS}

Alarcón, D. (2015). Educación en virtudes y valores formando el carácter y construyendo al ciudadano íntegro y libre. Orbis. Revista Cientifica Ciencias Humanas, 10(30), 48-59.

Alcocer, P. M., Rodríguez, A., y Arango, J. L. (2016). Formación docente para promover valores morales en la Universidad de Guayaquil. Revista Universidad y Sociedad, 8(2), 179-186.

Beltrán, O. A. (2005). Revisiones Sistemáticas de la Literatura. Revista Colombiana de Gastroenterología, 20(1), 60-69. Recuperado de http://www.scielo.org.co/pdf/rcg/v20n1/v20n1a09.pdf

Boroel, B. y Arámburo, V. (2016). El posicionamiento del y la docente ante la formación en valores en la educación superior. RIDE Revista Iberoamericana para la Investigación y el Desarrollo Educativo, 7(13), 463-482.

Cantú, P. (2018). Profesorado universitario: Emisor de valores éticos y morales en México. Revista Educación, 42(1), 1-24. doi: https://doi.org/10.15517/revedu.v42il.23479

Carrero, W. (2013). La educación en valores como fortalecimiento de la democracia. Revista de Ciencias Sociales (Ve), 19(3), 577-587.

Castro, D. y Díaz, M. (2015). Valores sociales y métodos de enseñanza: lo que deben mejorar los docentes universitarios desde la visión de los docentes. Humanidades Médicas, 15(3), 582-602.

Chapa, P. y Martínez, T. J. (2015). Valores Universitarios en los Jóvenes Estudiantes de la Facultad de Ciencias Políticas y Administración Pública, UANL. RIDE Revista Iberoamericana para la Investigación y el Desarrollo Educativo, 6(11), 773-786.

Del Salto Bello, M. (2015). Educación en valores: propuesta de una estrategia. Medisan, 19(11), 5018-5026. 
Escobar, J. P. (2013). Educar en Valores en el Siglo XXI: Una reflexión pedagógica más allá de la imposición y la costumbre. Revista Calidad en la Educación Superior, 4(2), 169-183

Estrada, O. (2012). El profesor ante la formación de valores. Aspectos teóricos y prácticos. Teoría de la Educación. Educación y Cultura en la Sociedad de la Información, 13(3), 240-267.

García, L., Ruiz, M. y García, M. (2011). Claves para la educación: actores, agentes y escenarios en la sociedad actual. Madrid, España: Narcea

Herrera, M. I. (2007). Los valores de los adolescentes, de sus padres y profesores, en función de que el contexto educativo sea monocultural o pluricultural. (Tesis doctoral). Universidad de Granada, Ceuta, España.

Hodelín, R. y Fuentes, D. (2014). El profesor universitario en la formación de valores éticos. Educación Médica Superior, 28(1), 115-126.

Latapí, P. (2001). Valores y Educación. Ingenierías, 4 (11) 59-69. Recuperado de http://www.ingenierias.uanl.mx/1 $1 /$ index.html

Martínez, M. (2006). Formación para la ciudadanía y educación superior. Revista Iberoamericana de Educación, 42, 85-102 Recuperado de https://rieoei.org/historico/documentos/rie42a05.pdf

Merino, P. (2007). Educación moral en la familia: desafíos y oportunidades. Rexe. Revista de Estudios y Experiencias en Educación, (11), 113-124.

Merma, G., Peiró i Gregòri, S. y Gavilán, D. (2013). Perspectivas sobre educación en valores en tiempos de crisis. Barataria. Revista Castellano-Manchega de Ciencias Sociales, (15), 151-160.

Moreno, M. C. y Ramírez, L. N. (Noviembre, 2018). Revisión de literatura sobre la enseñanza en valores en la educación superior. Tercer Congreso online internacional sobre desigualdad social, educativa y precarización en el siglo XXI. Universidad de Málaga, España. Recuperado de https://bit.ly/2XNBoBi

Organización para la Cooperación y el Desarrollo Económicos [OCDE]. (2017). Panorama de la Educación 2017. Recuperado de https://bit.ly/2IFuJRd

Palomer, L. y López, R. (2016). Medición de los valores éticos y morales enseñados en la carrera de Odontología de la Pontificia Universidad Católica de Chile, desde la apreciación docente. FEM: Revista de la Fundación Educación Médica, 19(2), 77-84.

Penas, S. (2008). Aproximación a los valores y estilos de vida de los jóvenes de 13 y 14 años de la provincia de a Coruña. (Tesis doctoral). Universidad de Santiago de Compostela, A Coruña, España.

Quijano, D., Lorenzo, O. y Alegre, C. (2016). Elección y preferencia de valores en estudiantes universitarios mexicanos. Profesorado. Revista de Curriculum y Formación de Profesorado, 20(2), 18-38.

Quintana, J. M. (1993). Pedagogía Familiar, Madrid: Narcea, Ediciones.

Reyes, G., Guevara, J. I. y Bonne, N. M. (2017). Formación de valores en futuros docentes ecuatorianos. Medisan, $21(4), 468-474$.

Sánchez-Meca, J. (2010). Cómo realizar una revisión sistemática y un meta-análisis. Aula Abierta, 38(2), 53-64. Recuperado de https://www.um.es/metaanalysis/pdf/5030.pdf

Secretaría de Educación Pública [SEP]. (2017). Principales cifras del Sistema Educativo Nacional 2017-2018. Recuperado de https://bit.ly/2GcV7PW

UNESCO. (1998). Conferencia Mundial sobre la Educación Superior. La Educación Superior en el siglo XXI: Visión y acción (1998). Recuperado de http://www.unesco.org/education/educprog/wche/declaration_spa.htm

Vera, M., Cornejo, R., Rivas, L., Calizaya, J., Zamora, O. y García, J. (2016). Significado de los valores profesionales en docentes y estudiantes de enfermería, UNMSM - 2014. Anales de la Facultad de Medicina, 77(3), 225-229.

\section{BY-NC-ND}

